\title{
Real-Time Video Road Sign Detection and Tracking Using Image Processing and Autonomous Car
}

\author{
F.A. Azis, P.S.G. Ponaseran, Zamani Md Sani, M.S.M. Aras, M. Nur Othman
}

\begin{abstract}
Detection and monitoring of real-time road signs are becoming today's study in the autonomous car industry. The number of car users in Malaysia risen every year as well as the rate of car crashes. Different types, shapes, and colour of road signs lead the driver to neglect them, and this attitude contributing to a high rate of accidents. The purpose of this paper is to implement image processing using the real-time video Road Sign Detection and Tracking (RSDT) with an autonomous car. The detection of road signs is carried out by using Video and Image Processing technique control in Python by applying deep learning process to detect an object in a video's motion. The extracted features from the video frame will continue to template matching on recognition processes which are based on the database. The experiment for the fixed distance shows an accuracy of $99.9943 \%$ while the experiment with the various distance showed the inversely proportional relation between distances and accuracies. This system was also able to detect and recognize five types of road signs using a convolutional neural network. Lastly, the experimental results proved the system capability to detect and recognize the road sign accurately.
\end{abstract}

Keywords: Convolutional neural network, image processing, real-time, road signs.

\section{INTRODUCTION}

Real-time Road Sign Detection and Tracking (RSDT) researches are growing rapidly in the Malaysian autonomous car industries. Real-time means that the actual time when the process or event occurs. When real-time and RSDT are combined, the system will detect and recognize the road signs while the car is moving on, and at the same time, it will alert the drivers if the system detected any road signs. The road sign detection and tracking is a computerized system which will detect and recognize the road sign from a video.

According to the Malaysian Institute of Road Safety (MIROS) [1], the number of accidents increased by each year from 476196 cases in 2014 to 489606 cases in 2015 and rose to 521466 in the year of 2016. The main contributor to these accidents caused by the failure of drivers to notice the road signs and neglecting the traffic rules. Neglecting road sign was also contributed to high rates of accidents. Drivers ignored the important road signs such as warning, prohibition, stop and mandatory signs while driving on road due to less attention while driving and confused with many types of road signs in Malaysia's road. Besides that, this RSDT

Revised Manuscript Received on September 14, 2019. Teknikal Malaysia Melaka, Melaka, Malaysia. (Email: fadilah@utem.edu.my)

Zamani Md Sani, Faculty of Electrical Engineering, Universiti Teknikal Malaysia Melaka, Melaka, Malaysia. (Email: zamanims@utem.edu.my )

Mohd Shahrieel Mohd Aras, Faculty of Electrical Engineering, Universiti Teknikal Malaysia Melaka, Melaka, Malaysia. (Email: shahrieel@utem.edu.my)

M. Nur Othman, Faculty of Mechanical and Manucfacturing Technology Engineering, Universiti Teknikal Malaysia Melaka, Melaka, Malaysia.( Email: muhammadnur@utem.edu.my)
Fadilah Abdul Azis, Faculty of Electrical Engineering, Universiti

system also could act as driving assistance for tourist who depends on the road sign while traveling to another country. RSDT system will interpret the road sign for the drivers and so that the drivers would not get confused.

The majority of the existing system used the method of image processing to detect and track the road signs. The image processing took the input image such as video's frame in the form of signal processing. Video is an electronic technology that capture, record, process, store, and transmit scenes in motion. Video is used in this system to obtain a better visual appearance of the road sign and to get ready the image to be measured for the features present.

The information source for this project is the road signs used on the street side. Road signs will provide important information regarding the conditions or circumstances of the road to drivers. Different types of road signs have been utilized on the road, which was different in shapes and colours that provide information like a warning, direction, prohibition, mandatory, and obligation of the roads. The system will detect road sign which stating the upcoming conditions of the road and provide with display and description about the signs. The RSDT system will assist drivers in having a safe and convenient journey. This paper presents a Haar cascade classifier for the detection process and convolutional neural network for recognition process for five types of road signs such as "stop", "speed bump", "t-junction", "no right turn" and "direction".

\section{PREVIOUS WORKS}

Previous research projects showed that a lot of techniques were introduced to achieve good results. Road signs could be detected and classified by using few features such as shape, colour, and pictogram.

In [2] used the method Support Vector Machine for the identification and recognition of road signs project. The author used the colour-based detection technique with Hue, Saturation and Intensity (HSI) and States that when there were changes in brightness, picture reflection and lightning, the Red, Green and Blue (RGB) colour space will unstable. The previous research's result using RGB is not accurate as HSI. SVM was used together with Gaussian kernels to recognize road signs.

In [3] researched for detection and recognition of road signs with bad visual. The author used Speeded Up Robust Features (SURF) algorithm for the detection system and Optical Character Recognition (OCR) for the recognition of the road signs. The maximally stable external regions and Canny detector is also proposed for the recognition process. The project achieved $92.18 \%$ of accuracy in detecting and recognizing the road signs.

Research on traffic symbol detection and recognition is carried out by [4]. The maximally stable external region has been used for the detection process based on the background colour. The traffic signs are separated based on their colour variously. Histogram of oriented gradient (HOG) method is used to compute the candidates that been detected and lastly Random Forest type of classifier is 


\section{REAL-TIME VIDEO ROAD SIGN DETECTION AND TRACKING USING IMAGE PROCESSING AND AUTONOMOUS CAR}

used for the classification method. The author stated that the accuracy of the detection and recognition increase as the car speed increases. Meanwhile, in [6] also applied the same technique as [4]. But, he applied colour separations method together with Bag of visual word algorithm on all frames for the detection of the blue and white panels of the road signs. The region of the images was classified by using two methods which is Naïve Bayes classifier and SVM. The dataset was obtained from internet source. The accuracy for detection is $90.18 \%$ while for recognition is $87.50 \%$.

In [5] obtained quicker and accurate results by using SVM. This was because it is not too prone in overfitting. However, SVM is not suitable for a critical time. Besides that, the author used Histogram of oriented gradient (HOG) together with distance transform (DTs) for effective classification of the road sign. Random forest classifier was used to enhance the performance of the system, and about 5000 images were trained for the dataset. The author claimed that the more dataset provided, the more accurate the detection and recognition result will be.

In [7] used the method of Hue, Saturation and Value (HSV). Based on that research, HSV is able to detect text features of the road sign successfully. HSV is better than RGB because it is more equivalent to the perception of human seeing the object. Using RGB method the accuracy of the detection is less, this was because of the lighting changes when the process was running. For the larger numbers of features, the maximally stable external region method is applied, and the OCR method was used for the recognition process. The system produces a better detection result with the accuracy of $90.18 \%$ and $87.50 \%$ accuracy for recognition results.

In [8] did a real-time traffic sign detection and classification by using colour images as an input image. The maximally stable external region is used to extract the road signs. The HOG is used together with SVM classifier for the classification process by filtering out the false negative. The author proposed the Convolutional Neural Network (CNN) to classify the detected road signs, and this method produced an efficiency of 20 times even quicker.

Lastly, more processing time is taken by [9] because of larger neural networks. However, the neural network does not require to reprogram. The author did colour segmentation by using hybrid colour segmentation and robust custom features. For the road sign classification, an Artificial Neural Network method is proposed. Based on previous works, CNN method has been chosen for this project because it produces high recognition accuracy.

\section{METHODOLOGY}

Image processing and Artificial Intelligence (AI) techniques are being implemented in this project with python application to detect and classify the road signs. Fig. 1 shows some examples of road signs considered in this work while Fig. 2 shows the overview of the system. The road sign detection process developed by using the technique of image acquisition, collecting dataset, and Haar cascade classifications.

Image processing is the first process of image acquisition. The image created for dataset is included as image acquisition. The webcam is connected to a personal computer inside a car and then the webcam is fixed in the middle of the dashboard.

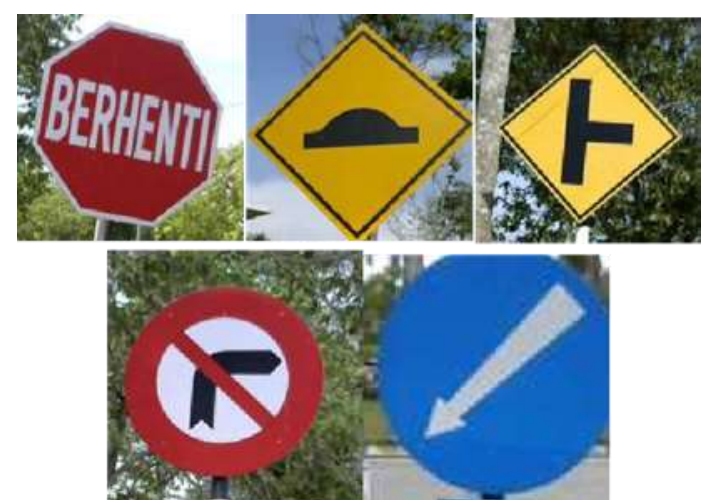

Fig. 1. Examples of road signs considered in this work

Training and Testing Process Field Application

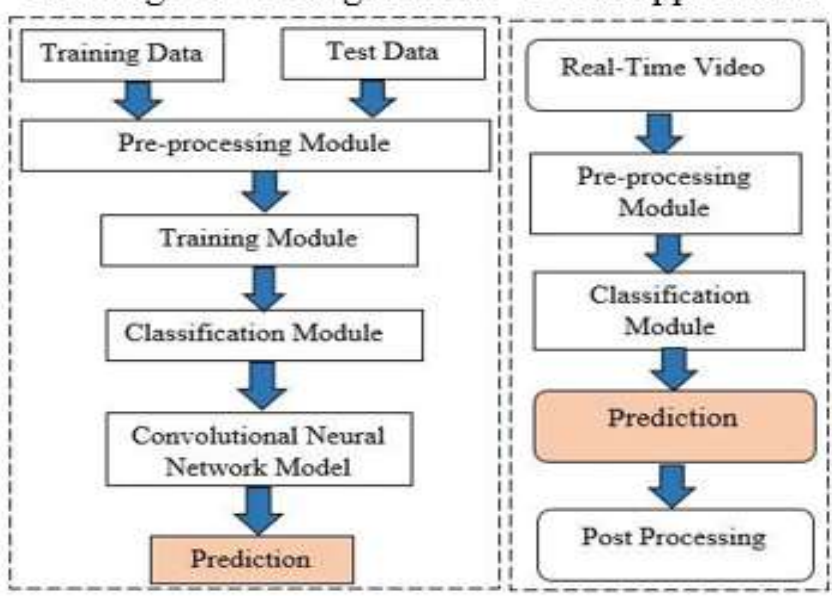

Fig. 2. Overview of the system

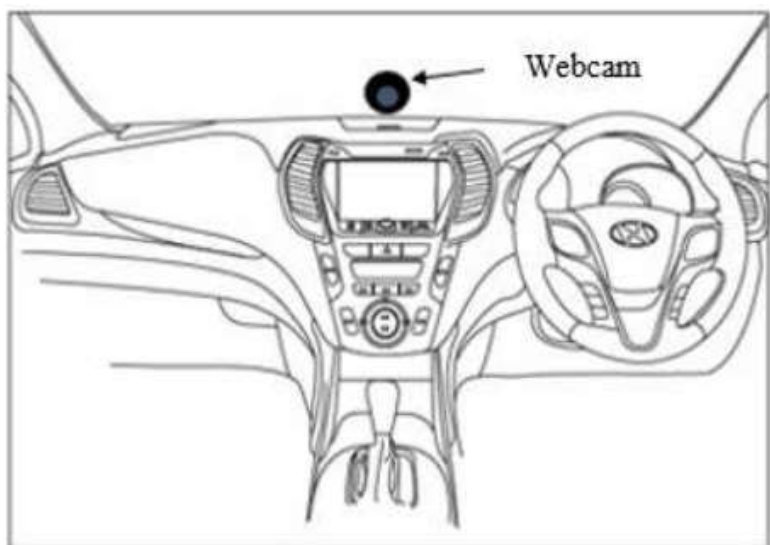

Fig. 3. Position of webcam inside an autonomous car

The position of a webcam is fixed in the middle of the autonomous car as in Fig. 3, so that it will able to detect, classify, and recognize road signs on the left and right side correctly. The image is taken on good weather and lighting. Besides that, the car is driven in the speed of not more than 60 kilometres per hour during the real-time video process.

The total number of images used as a dataset in this project is 397 images. 189 images consist of negative images and 208 images are positive. The negative images consist of background images of road sign such as buildings, trees, cars, and roads while positive images consist of different types of road signs. Negative images are the images that would not be detected during the detection process and positive images are road sign images.

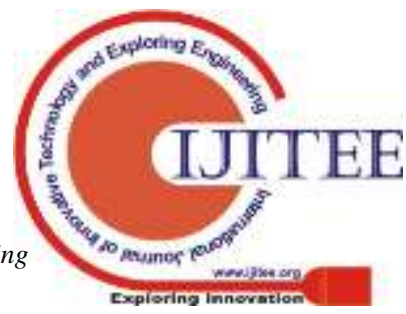


OpenCV provides Haar cascade classifier which is an effective technique for road sign detection. Haar cascade algorithm will produce high detection accuracy and lower false-positive rates. Haar cascade classifier trained positive and negative images by extracting features of Haar from all the images. Both negative and positive images are located in a different folder. A vector file is created using objectmarker utility. Objectmarker.exe is run and the positive images windows will pop out to mark the road signs.

The road sign is marked with a rectangle bounding box and the tool will automatically save the position and size of the rectangle. Vector of positive images is created with that parameter of 100000 numbers of positive files and with the width and height object is 24 pixels. Finally, haar training is run by providing the number of positive and negative samples and the XML file is created. The file is then run in pycharm using python to get the result in real-time video. More positive images must be provided if the system could not detect the road signs correctly and negative images if the system detected any objects other than road signs.

In road sign classification and recognition process, the dataset is prepared for Convolutional Neural Network training and classification. Five types of road signs were collected as the main data. The selected road signs were Stop, No Turn Right, Direction, T-junction and Speed Bump signs. These five types of road signs are normally found in Malaysia's road. 3000 images were collected as data set for each road sign, where 15000 total images for five types of road signs. The dataset was constructed automatically by capturing images using python. The dataset images consist of colour images with the resolution of 170 by 170 dimensions and in the format of JPG file. Images in JPG format are compatible for training.

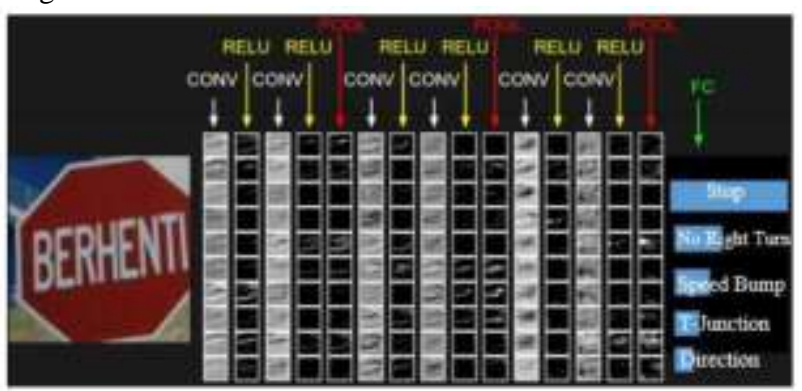

Fig. 4. Architecture of layer for stop sign

Convolutional Neural Network is one of the Artificial Intelligence (AI) method used in the project. The dataset of 15000 images of the road signs were trained by building a CNN that will predict each type of road signs. The CNN is built by using Keras deep learning library in python to classify each of the road signs and it is selected because it required minimal prepossessing. Then, the road sign images are filtered by using three types of layers which include input, hidden and output layers.

The input layer is a convolutional layer where the neurons apply the operation of convolution to the input. The input images are basically in a 2-dimensional array of pixels. The images are trained for 50 number of epochs, batch size of 32 with the dimension $32 \mathrm{x}$ $32 \times 3$. The higher the value of the epochs number, the higher the accuracy of detecting and recognizing the road signs. But, higher epochs number required more time to train the dataset. The dataset is divided into 32 number of batch during the training process. The filter size consists of 3 channels in coloured images. The features are filtered in convolutional layers.

The hidden layer which is pooling layer will scale down the size of inputs into half. The width and height is reduced while the depth remains the same. The features will be similar to the input layer but smaller in size. The ReLu layer later converts pixel's negative value by zero. The output layer is a fully connected layer, where high-level reasoning is completed. It connecting all neuron in a layer and the comparison will start to produce the outputs. The output of the training and classification process will be produced in a model file for the next step which is road sign recognition. Fig. 4 shows the architecture layer for the stop sign.

Convolutional Neural Network Equations

Layers Equations

$\mathrm{N}$ x N = square neuron layers

$\mathrm{m} \times \mathrm{m}=$ Filter $(\mathrm{w})$

$(N-m+1) \times(\mathrm{N}-\mathrm{m}+1)=$ output layers

$x_{i j}^{l}=\sum_{a=0}^{m-1} \cdot \sum_{b=0}^{m-1} \omega a b y_{(i+a)(j+b)}^{l-1}$

\section{Max-Pooling Layers}

$K \times K=$ Region

$$
(N / K) \times(N / K)=\text { Layers }
$$

Road sign recognition is the final part of the project, which will generate the results that have been compared with the stored dataset. In the recognition process, label from the mapping process is renamed with the original name or function of the road signs. The name will be displayed if the system successfully detects and recognize the road sign. The output files of haar training and CNN training are then extracted by using python. The scale factor is adjusted to 1.6 pixels. The detection of a road sign will be accurate if the factor is high. Minimum neighbours is increased to 60 pixels and the minimum size is set as $60 \times 60$ pixels. Minimum size is controlling the detection of the road sign. If the parameter of road sign appears in the frame is smaller than the minimum size, the system will ignore the road sign. The system is run with a webcam connected with a personal computer in a moving car. In this phase, if the system detected and recognized the road signs, it will construct a boundary box with the function and accuracy of the road signs.

The experiments had been carried out to analyze the accuracy of road sign detection and recognition. For each of the experiments, five times of repeatability test was conducted to analyze the accuracy of the road signs. Three types of experiments are conducted for the analysis; the experiment of detection and recognition on a constant distance of 3 metre, experiment of detection and recognition on various distances such as the distance fixed is 4 metre, 5 metre, 6 metre, and experiment of detection and recognition on different light intensity.

\section{RESULTS AND DISCUSSION}

For this project, the system is implemented by using python 3.6, running on a $3 \mathrm{~GB}$ ram Intel core i5 personal computer with 5MP webcam. Overall system performance will be discussed on the result of evaluation based on the accuracy of the detection and classification process from a fixed distance, various distance, and different light intensity. The results are tabulated in the tables with the value of True Positive, True Negative, False Positive, False Negative, error rate, and accuracy. The overall evaluation of the system is collected using calculation theory by referencing formulas.

- $\quad$ True Positive $=$ Video frame with road sign is precisely detected and recognised as having road sign by the experiment.

- $\quad$ True Negative = Video frame without road sign is precisely detected and recognised as having no road sign by the experiment.

- $\quad$ False Positive $=$ Video frame without road sign is incorrectly detected and recognised as having road sign by the experiment. 


\section{REAL-TIME VIDEO ROAD SIGN DETECTION AND TRACKING USING IMAGE PROCESSING AND AUTONOMOUS CAR}

- $\quad$ False Negative $=$ Video frame with road sign is incorrectly detected and recognised as having no road sign by the experiment.

- Error rate, $\square \varepsilon=\frac{\text { Total no. of false positive }}{\left(\begin{array}{l}\text { Total no. of false positive }+ \\ \text { Total no. of true positive }\end{array}\right)}$;

- Mean of total error rate $=\frac{\text { Total error rate }}{\text { Total no. of test }}$;

- Accuracy $(\%)=100-$ Mean of total error rate.

For the experiment of detection and recognition on a constant distance, the distance is fixed at 3 metre between a moving car to a road sign with the driving speed below $60 \mathrm{~km} / \mathrm{h}$, and the frame rate is $30 \mathrm{fps}$. The experiment is tested with five times of repeatability for each road signs. Table I shows the accuracy of road signs from a distance of 3 metre. Fig. 5 shows the graph of accuracy versus types of a road sign.

The process of repeating a test enables to identify the error and accuracy in the road sign detection and classification process. The direction sign accuracy is the highest among the other road sign which is $99.9996 \%$, whereas the lower accuracy obtained from the experiment is $99.9879 \%$ for the speed bump sign. Thus the accuracy of the detection and classification of road sign could not not achieve $100 \%$ due to error rate obtained during the experiment. The factors that cause the rate of error to increase are the intensity of the light and the resolution of the camera. Fig. 6 shows the detection and recognition for five types of road signs.

Table- I: Accuracy of road signs from distance 3 metre

\begin{tabular}{|l|c|c|c|c|c|c|}
\hline Road Sign & Test & $\begin{array}{c}\text { Average } \\
\text { Trae } \\
\text { Positive }\end{array}$ & $\begin{array}{c}\text { Average } \\
\text { False } \\
\text { Positive }\end{array}$ & $\begin{array}{c}\text { Average } \\
\text { False } \\
\text { Negative }\end{array}$ & $\begin{array}{c}\text { Average } \\
\text { Error } \\
\text { Rate }\end{array}$ & $\begin{array}{c}\text { Accuracy } \\
(\%)\end{array}$ \\
\hline Stop & 5 & 0.9957 & 0.0056 & 0.0043 & 0.0046 & 99.9954 \\
\hline Speed Bump & 5 & 0.9893 & 0.0119 & 0.0107 & 0.0121 & 99.9879 \\
\hline T-Jmetion & 5 & 0.9936 & 0.0094 & 0.0063 & 0.0079 & 99.9921 \\
\hline No Right Tum & 5 & 0.9977 & 0.00382 & 0.0022 & 0.0032 & 99.9968 \\
\hline Direction & 5 & 0.9999 & 0.00042 & 0.0001 & 0.0004 & 99.9996 \\
\hline Average & & 0.99524 & 0.006228 & 0.00472 & 0.00564 & 99.99436 \\
\hline
\end{tabular}

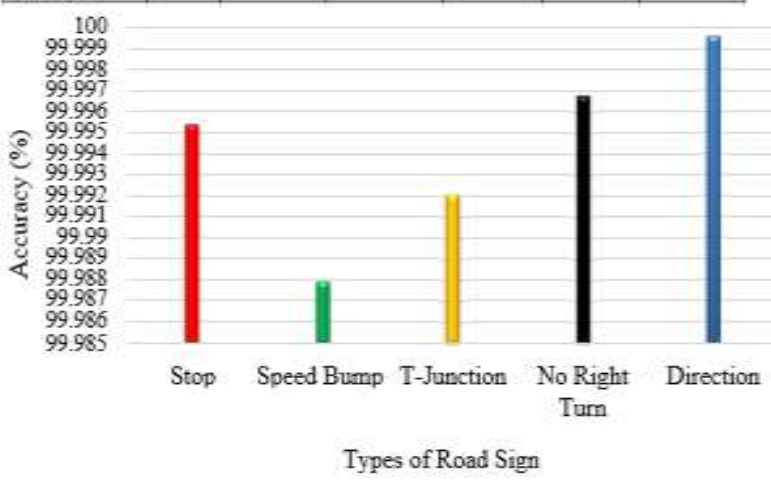

Fig. 5. Graph of accuracy vs types of road sign

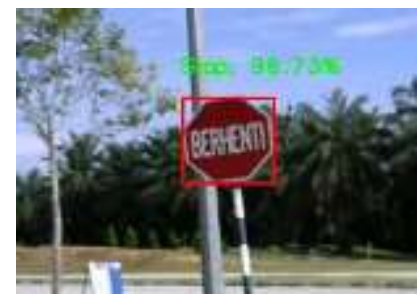

a)

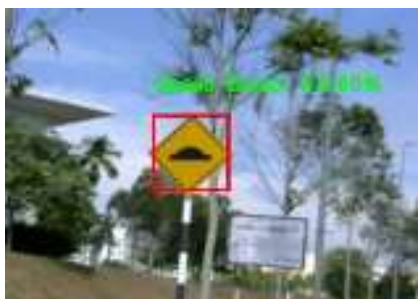

b)

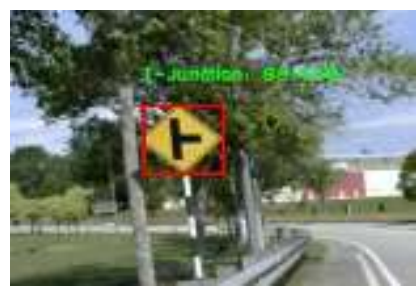

c)

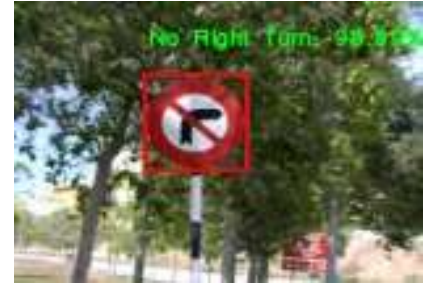

d)

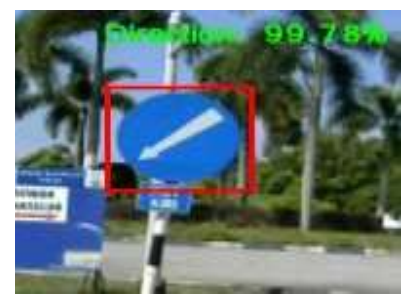

e)

Fig. 6. Detection and recognition for 5 types of road signs: a) Stop sign, b) Speed bump sign, c) T-Junction sign, d) No right turn sign and e) Direction sign

For the experiment of detection and recognition on a various distance, the distance fixed is 4 metre, 5 metre and 6 metre between a moving car to a road sign with the driving speed below $60 \mathrm{~km} / \mathrm{h}$ and the frame rate is 30fps. Experiment is tested with five times of repeatability for each road signs. Table II shows the accuracy of road signs from the distance of 4 metre, Table III shows the accuracy of road signs from the distance of 5 metre and Table IV shows the accuracy of road signs from the distance of 6 metre.

The data as shown in Table II is the result obtained after five times of repeatability for each road sign for a distance of 4 metre between road signs and car while driving. The highest accuracy of detection and recognition for this experiment is $99.9915 \%$ which is the direction sign. The lowest is T-Junction sign with the accuracy 99.9807\%. In Table III, shows the experiment of road sign detection and recognition for the distance of 5 metre. Direction sign gained the highest accuracy with 99.9876 and T-junction with the lowest accuracy which is $99.9719 \%$. Lastly, road sign detection and recognition for a distance of 6 metre having $99.9779 \%$ of the highest accuracy for the direction sign and the lowest accuracy is speed bump sign with the accuracy of $99.9663 \%$ are shown in Table IV.

Table- II: Accuracy of road signs from distance of 4 metre

\begin{tabular}{|l|c|c|c|c|c|c|}
\hline Road Sign & Test & $\begin{array}{c}\text { Average } \\
\text { True } \\
\text { Positive }\end{array}$ & $\begin{array}{c}\text { Average } \\
\text { False } \\
\text { Positive }\end{array}$ & $\begin{array}{c}\text { Average } \\
\text { False } \\
\text { Negative }\end{array}$ & $\begin{array}{c}\text { Average } \\
\text { Error } \\
\text { Rate }\end{array}$ & $\begin{array}{c}\text { Accuracy } \\
\text { (\%) }\end{array}$ \\
\hline Stop & 5 & 0.98372 & 0.01496 & 0.0163 & 0.0149 & 99.985 \\
\hline Speed Bump & 5 & 0.98144 & 0.01682 & 0.0186 & 0.0168 & 99.9831 \\
\hline T-Junction & 5 & 0.98004 & 0.0193 & 0.01996 & 0.0193 & 99.9807 \\
\hline No Right Tunn & 5 & 0.9856 & 0.01572 & 0.0164 & 0.0157 & 99.9843 \\
\hline Direction & 5 & 0.99094 & 0.00854 & 0.00906 & 0.0085 & 99.9915 \\
\hline Average & & 0.984348 & 0.015068 & 0.01606 & 0.01504 & 99.98492 \\
\hline
\end{tabular}

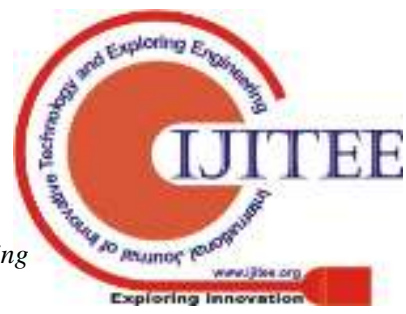


Table- III: Accuracy of road signs from distance of 5 metre

\begin{tabular}{|l|c|c|c|c|c|c|}
\hline Road Sign & Test & $\begin{array}{c}\text { Average } \\
\text { True } \\
\text { Positive }\end{array}$ & $\begin{array}{c}\text { Average } \\
\text { False } \\
\text { Positive }\end{array}$ & $\begin{array}{c}\text { Average } \\
\text { False } \\
\text { Negative }\end{array}$ & $\begin{array}{c}\text { Average } \\
\text { Error } \\
\text { Rate }\end{array}$ & $\begin{array}{c}\text { Accuracy } \\
(\%)\end{array}$ \\
\hline Stop & 5 & 0.9777 & 0.2082 & 0.0223 & 0.02084 & 99.9792 \\
\hline Speed Bump & 5 & 0.97196 & 0.0267 & 0.02804 & 0.02672 & 99.9733 \\
\hline T-Juction & 5 & 0.97052 & 0.02804 & 0.02948 & 0.02806 & 99.9717 \\
\hline No Right Turn & 5 & 0.97694 & 0.02248 & 0.02306 & 0.02164 & 99.9783 \\
\hline Direction & 5 & 0.9856 & 0.01238 & 0.0144 & 0.0124 & 99.9876 \\
\hline Average & & 0.976544 & 0.05956 & 0.02346 & 0.02193 & 99.97802 \\
\hline
\end{tabular}

Table- IV: Accuracy of road signs from distance of 6 metre

\begin{tabular}{|l|c|c|c|c|c|c|}
\hline Road Sign & Test & $\begin{array}{c}\text { Average } \\
\text { True } \\
\text { Positive }\end{array}$ & $\begin{array}{c}\text { Average } \\
\text { False } \\
\text { Positive }\end{array}$ & $\begin{array}{c}\text { Average } \\
\text { Fulse } \\
\text { Nezative }\end{array}$ & $\begin{array}{c}\text { Average } \\
\text { Error } \\
\text { Rate }\end{array}$ & $\begin{array}{c}\text { Aceuracy } \\
\text { (6) }\end{array}$ \\
\hline Stop & 5 & 0.97238 & 0.0278 & 0.02762 & 0.0278 & 99.9722 \\
\hline Speed Bump & 5 & 0.96682 & 0.03366 & 0.03318 & 0.03366 & 99.9663 \\
\hline T-Junction & 5 & 0.96686 & 0.03298 & 0.03314 & 0.03298 & 99.967 \\
\hline No Right Tum & 5 & 0.97134 & 0.02876 & 0.02866 & 0.02876 & 99.9712 \\
\hline Direction & 5 & 0.97746 & 0.02208 & 0.02254 & 0.02208 & 99.9779 \\
\hline Average & & 0.970972 & 0.029056 & 0.02903 & 0.02906 & 99.97092 \\
\hline
\end{tabular}

Graph of accuracy vs distance is constructed to have a clear picture on the result of total accuracy for each experiment. The graph shows that the accuracy of road sign detection and recognition is decreasing for all road signs as the distance is increasing. Factors that affect the reducing of accuracy is the resolution of the webcam. The accuracy of RSDT can be increased by using webcam with better resolution. Fig. 7 shows the graph of accuracy vs distance.

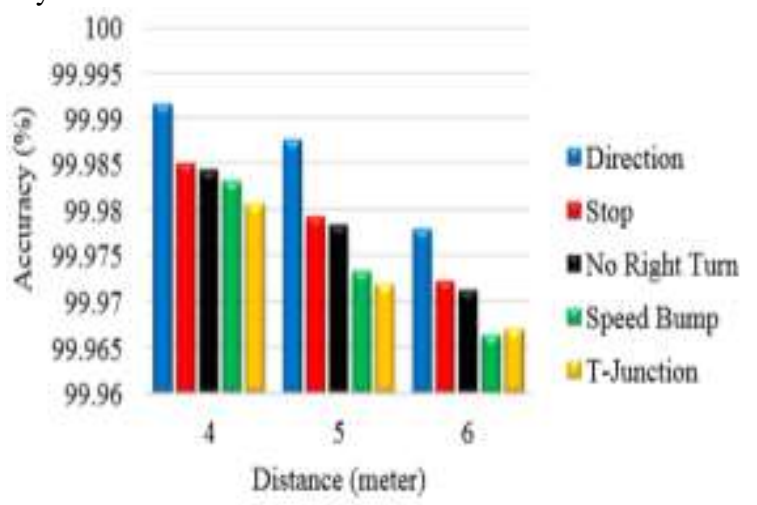

Fig. 7. Graph of accuracy vs distance

The experiment of detection and recognition on different light intensity is conducted on morning and afternoon with the driving speed below $60 \mathrm{~km} / \mathrm{h}$ and the frame rate is $30 \mathrm{fps}$. The distance is fixed at 3 metre from road sign to the car. An environment with low light intensity produces less accurate result because of the dullness on road signs. High light intensity makes an environment brighter where it will make the road sign's colours reflected and even brighter. Table $\mathrm{V}$ shows the accuracy of road signs on morning (58864 lux) and Table VI shows the accuracy of road signs on the afternoon (97823 lux).

The result tabulated in the table shows that the total accuracy on the morning is $99.9934 \%$ while in the afternoon is $99.9966 \%$. The accuracy in the afternoon is higher because of the higher value of light intensity. Bright light environment makes the road signs even clearer in real-time video and easy to detect and recognize. The light source on the morning is not enough to make the road sign to look clear. Fig. 8 shows the graph of accuracy vs different light intensity
Table- V: Accuracy of road signs on morning

\begin{tabular}{|l|c|c|c|c|c|c|}
\hline Road Sign & Test & $\begin{array}{c}\text { Average } \\
\text { True } \\
\text { Positive }\end{array}$ & $\begin{array}{c}\text { Average } \\
\text { False } \\
\text { Positive }\end{array}$ & $\begin{array}{c}\text { Average } \\
\text { False } \\
\text { Negative }\end{array}$ & $\begin{array}{c}\text { Average } \\
\text { Error } \\
\text { Rate }\end{array}$ & $\begin{array}{c}\text { Accuracy } \\
(\%)\end{array}$ \\
\hline Stop & 5 & 0.99634 & 0.0035 & 0.00366 & 0.0035 & 99.9965 \\
\hline Speed Bump & 5 & 0.9913 & 0.00862 & 0.0087 & 0.00862 & 99.9914 \\
\hline T-Junction & 5 & 0.99154 & 0.00816 & 0.00846 & 0.00816 & 99.9918 \\
\hline No Right Turn & 5 & 0.9931 & 0.0068 & 0.00694 & 0.00676 & 99.9931 \\
\hline Direction & 5 & 0.99406 & 0.00572 & 0.00594 & 0.00572 & 99.9943 \\
\hline Average & & 0.99326 & 0.006552 & 0.00674 & 0.00655 & 99.99342 \\
\hline
\end{tabular}

Table- VI: Accuracy of road signs on afternoon

\begin{tabular}{|l|c|c|c|c|c|c|}
\hline Road Sign & Test & $\begin{array}{c}\text { Average } \\
\text { True } \\
\text { Positive }\end{array}$ & $\begin{array}{c}\text { Average } \\
\text { False } \\
\text { Positive }\end{array}$ & $\begin{array}{c}\text { Average } \\
\text { False } \\
\text { Negative }\end{array}$ & $\begin{array}{c}\text { Average } \\
\text { Error } \\
\text { Rate }\end{array}$ & $\begin{array}{c}\text { Accuracy } \\
\text { (\%) }\end{array}$ \\
\hline Stop & 5 & 0.9993 & 0.00058 & 0.0007 & 0.00058 & 99.9994 \\
\hline Speed Bump & 5 & 0.99396 & 0.00552 & 0.00604 & 0.00552 & 99.9945 \\
\hline T-Junction & 5 & 0.99414 & 0.00568 & 0.00586 & 0.00568 & 99.9943 \\
\hline No Right Turn & 5 & 0.99504 & 0.00472 & 0.00496 & 0.00472 & 99.9953 \\
\hline Direction & 5 & 0.99962 & 0.0004 & 0.00038 & 0.0004 & 99.9996 \\
\hline Average & & 0.996412 & 0.00338 & 0.00359 & 0.00338 & 99.99662 \\
\hline
\end{tabular}

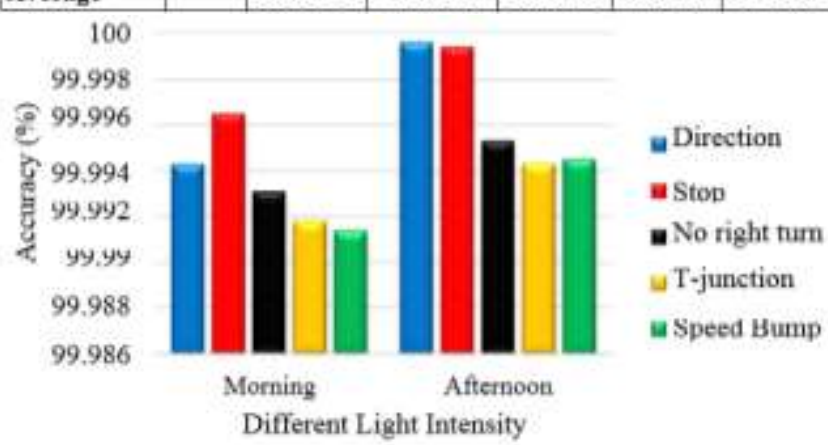

Fig. 8. Graph of accuracy vs different light intensity

\section{CONCLUSION}

The real-time video road sign detection system is successfully designed and implemented by using the image processing method and the autonomous car. All five different types, of road signs, are classified successfully. Lastly, the result enabled us to analyze and improve the classification accuracy of road sign detection by using convolutional neural network method. The first experiment for the fixed distance shows an accuracy of $99.9943 \%$ while the experiment with the various distance confirm that the accuracy decreased as the distance increases. For the distance of 4 metre from the road sign to camera the accuracy is $99.9849 \%$, while accuracy for 5 metre is $99.9780 \%$ and for 6 metre is $99.9710 \%$. For the light intensity on the morning, the accuracy is $99.9934 \%$, and on the afternoon the accuracy is $99.9966 \%$.

As for the recommendation, by using a camera with a better resolution it will produce a better accuracy as the distance between the road sign and the car is increasing. Furthermore, the high resolution camera able to detect and recognize the road sign while the intensity of the light is low. The second recommendation is to provide more dataset images of the road signs from different views to make the detection and recognition process faster and accurate and can reduce the error rate. Last recommendation is to establish the system together with hardware application and equip with global positioning system for an autonomous car. 


\section{REAL-TIME VIDEO ROAD SIGN DETECTION AND TRACKING USING IMAGE PROCESSING AND AUTONOMOUS CAR}

\section{ACKNOWLEDGMENT}

The author would like to acknowledge the funding support received from Centre of Research and Innovation Management (CRIM) and Faculty of Electrical Engineering from Universiti Teknikal Malaysia Melaka (UTeM).

\section{REFERENCES}

1. Malaysian Institute of Road Safety Research (MIROS), Accident and death rates for the year 2014, 2015 and 2016 in Malaysia. Selangor: MIROS.

2. S. Maldonado-Bascon, S. Lafuente-Arroyo, Gil-Jimenez, H. Gomez-Moreno, and F. Lopez-Ferreras, "Road-sign detection and recognition based on support vector machines," IEEE Trans. Intell. Transp. Syst., 8(2), 2007, pp. 264-278.

3. D. S. Solanki and Gireesh Dixit, "Traffic sign detection and recognition using feature based and OCR method," Proc. IJRSET, 2(2), 2002, pp. 32-40.

4. J. Greenhalgh and M. Mirmehdi, "Traffic sign recognition using MSER and random forests," IEEE 20th European Signal Processing Conference, 2012, pp. 19351939.

5. F. Zaklouta and B. Stanciulescu, "Real-time traffic-sign recognition using tree classifiers," IEEE Trans. Intell. Transp. Syst., 13(4), 2012, pp. 1507-1514.

6. A. Gonzalez, L. M. Bergasa, and J. J. Yebes, "Text detection and recognition on traffic panels from streetlevel imagery using visual appearance," IEEE Trans. Intell. Transp. Syst., 15(1), 2014, pp. 228-238.

7. J. Greenhalgh and M. Mirmehdi, "Recognizing text based traffic signs," Trans. Intell Transp. Syst., 16(3), 2014, pp. 1360-1369.

8. Y. Yang, H. Luo, H. Xu, and F. Wu, "Towards real-time traffic sign detection and classification," Trans. Intell. Transp. Syst, 17(7), 2015, pp. 2022-2031.

9. K. Islam and R. Raj, "Real time (vision based) road sign recognition using an artificial neural network," Sensors, 17(4), 2017, pp. $1-32$

\section{AUTHORS PROFILE}

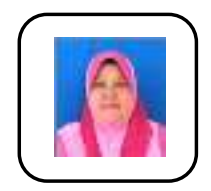

F.A. Azis is a lecturer of Faculty of Electrical Engineering in Universiti Teknikal Malaysia Melaka (UTeM). She has a Bachelor of Mechatronics Engineering from International Islamic University Malaysia and a Master in Mechatronics Engineering from the University of Siegen, Germany. Her research interest includes the areas of Nonlinear Control and Underwater Technology. She is currently working on the research with the unmanned aerial vehicles (UAV) system.

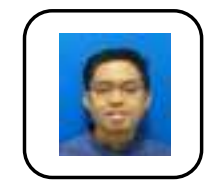

Zamani Md Sani received his Bachelor and Master in Electrical and Electronics Engineering from University Sains Malaysia in 2000 and 2009. His research interest is image processing. Currently, he is pursuing his Ph.D. degree from MMU and also a senior lecturer at Universiti Teknikal Malaysia Melaka.

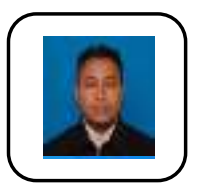

Dr. Mohd Shahrieel b Mohd Aras is Associate Professor at Faculty of Electrical Engineering, Universiti Teknikal Malaysia Melaka UTeM. His current research is focusing on control system design of underwater technology. His primary interests related to underwater robotics and Artificial Intelligence.

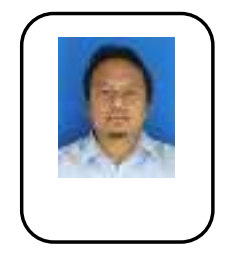

M. Nur Othman is a lecturer at Faculty of Engineering Technology, Universiti Teknikal Malaysia Melaka (UTeM). He has a Bachelor of Engineering (Mechanical-Automotive). Hons. in 2006 from International Islamic University Malaysia (IIUM) and Master of Engineering (Mechanical) in 2016 from University of Malaya. His research interest includes NVH, HVACR, CAE, Renewable Energy and Underwater Research. 